\title{
Packing Constant in Musielak-Orlicz Sequence Spaces Equipped with the Luxemburg Norm
}

\author{
HENRYK HUDZIK, CONGXIN WU and YINING YE
}

\begin{abstract}
A morè precise formula for the Kottman parameter $D(X)$ connected with the packing constant $\Lambda(X)$ in such a way that $\Lambda(X)=$ $D(X) /(2+D(X))$ for a Banach space $X$, in the case when $X$ is a MusielakOrlicz sequence spaces $l^{\varphi}$, is given. As a corollary, packing constant of the Nakano space $l^{\left(p_{i}\right)}$, where $1 \leq p_{i}<+\infty$ for any $i=1,2, \ldots$, is computed. This generalizes the results of [2] for $l^{p}$ spaces. It is also proved that $\Lambda\left(l^{\varphi}\right)=$ $\Lambda\left(h^{\varphi}\right)$.
\end{abstract}

\section{INTRODUCTION}

In the sequel $\mathbf{N}, \mathbf{R}, \mathbf{R}_{+}$and $\mathbf{R}_{+}^{\mathbf{e}}$ stand for the set of natural numbers, the set of reals, the set of positive reals and for the interval $[0,+\infty]$, respectively. The space of all real sequences $x=\left(x_{i}\right)_{i=1}^{\infty}$ is denoted by $l^{0}$. A map $\varphi: \mathbf{R} \rightarrow \mathbf{R}_{+}^{e}$ is said to be an Orlicz function if $\varphi$ is convex, even, vanishing and continuous at 0 , left-hand side continuous on the whole $\mathbf{R}_{+}$, and not identically equal to zero (see [9], [10] and [11]).

A sequence of $\varphi=\left(\varphi_{i}\right)_{i=1}^{\infty}$ of Orlicz functions $\varphi_{i}$ is called a Musielak-Orlicz function.

1991 Mathematics Subject Classification: 46E30

Editorial Complutense. Madrid, 1994. 
Given a Musielak-Orlicz function $\varphi$ we define on $l^{0}$ a convex modu$\operatorname{lar} I_{\varphi}$ by

$$
I_{\varphi}(x)=\sum_{i=1}^{\infty} \varphi_{i}\left(x_{i}\right) \quad\left(\forall x=\left(x_{i}\right)_{i=1}^{\infty} \in l^{0}\right)
$$

A Musielak-Orlicz space generated by $\varphi$ is defined by

$$
l^{\varphi}=\left\{x \in l^{0}: I_{\varphi}(\lambda x)<+\infty \text { for a certain } \lambda>0\right\} .
$$

The functional

$$
\|x\|_{\varphi}=\inf \left\{\lambda>0: I_{\varphi}(x / \lambda) \leq 1\right\}
$$

is a norm in $l^{\varphi}$ (called the Luxemburg norm) and the couple $\left(l^{\varphi},\|\|_{\varphi}\right)$ is a Banach space (see [9]). We denote by $h^{\varphi}$ the subspace of $l^{\varphi}$ being the closure in $l^{\varphi}$ of all sequences in $l^{\circ}$ with finite number of coordinates different from zero. This subspace will be considered with the norm \|\|$_{\varphi}$ induced from $l^{\varphi}$. In the case when all functions $\varphi_{i}$ which define $\varphi$ are finite-valued (i.e. $\varphi_{i}$ are continuous functions) we have simply

$$
h^{\varphi}=\left\{x \in l^{0}: I_{\varphi}(\lambda x)<+\infty \text { for any } \lambda>0\right\} \text {. }
$$

We say a Musielak-Orlicz function $\varphi$ satisfies the $\delta_{2}^{0}$-condition if there are positive constants $a, K$, a natural number $m$ and a sequence $\left(C_{i}\right)_{i=1}^{\infty} \subset \mathbf{R}_{+}^{e}$ such that $\sum_{i=m}^{\infty} C_{i}<+\infty$ and for any $i \in \mathbf{N}$ and $u \in \mathbf{R}$ satisfying $\varphi_{i}(u) \leq a$ there holds

$$
\varphi_{i}(2 u) \leq K \varphi_{i}(u)+C_{i}
$$

If a Musielak-Orlicz function $\varphi$ satisfies the $\delta_{2}^{0}$-condition with $m=1$ we say that $\varphi$ satisfies the $\delta_{2}$-condition (see [4], [6] and [11]).

For any Musielak-Orlicz function $\varphi, h^{\varphi}$ coincides with $l^{\varphi}$ if and only if $\varphi$ satisfies the $\delta_{2}^{0}$-condition (see [5]). 
For any Banach space $X$ denote by $B(X)$ and $S(X)$ the unit ball and the unit sphere of $X$, respectively. The unit ball in $X$ centered at $x \in X$ and with radius $r>0$ denote by $B_{X}(x, r)$.

The packing constant $\Lambda(X)$ of a Banach space $X$ is defined by

$$
\begin{array}{r}
\Lambda(X)=\sup \left\{r>0: \exists\left(x^{n}\right)_{n=1}^{\infty} \subset B(X),\left\|x^{m}-x^{n}\right\| \geq 2 r\right. \\
\text { for } \left.m \neq n, \text { and } \bigcup_{n=1}^{\infty} B_{X}\left(x^{n}, r\right) \subset B(X)\right\}
\end{array}
$$

(see [12]). Kottman [8] proved that for any Banach space $X$ we have $\Lambda(X)=D(X) /(2+D(X))$, where

$$
D(X)=\sup _{\left(x^{n}\right) \subset S(X)} \inf _{m \neq n}\left\|x^{m}-x^{n}\right\| .
$$

It is well known that $D\left(l^{p}\right)=2^{1 / p}$ for $1 \leq p<+\infty$ and $D\left(l^{\infty}\right)=2$ (see [12]). Moreover, Cleaver [3] obtained some lower and upper estimations for the packing constant of Orlicz sequence (as well as function) spaces and he pointed out that these estimations give an exact formula for the packing constant in a special class of Orlicz spaces, i.e. for Orlicz spaces such that $\|x\|_{\varphi}=\varphi^{-1}\left(I_{\varphi}(x)\right)$ for any $x \in l^{\varphi}$ (respectively for any $x \in L^{\varphi}$ ). However, Zaanen [15] pointed out that this class of Orlicz spaces reduces only to $l^{p}$ (respectively $L^{p}$ ) spaces (see also Wnuk [13]). Next, Ye [14] obtained a simpler formula for $D\left(l^{\varphi}\right)$, where $\varphi$ is an Orlicz function (i.e. all functions $\varphi_{i}$ in the definition of $\varphi$ are the same). Namely, he has proved that for any finite-valued Orlicz function $\varphi$ which satisfies the $\Delta_{2}$-condition at zero, we have

$$
D\left(l^{\varphi}\right)=\sup _{x \in S(l \varphi)}\left\{c_{x}>0: I_{\varphi}\left(x / c_{x}\right)=\frac{1}{2}\right\} .
$$

In this paper we obtain an analogue for Musielak-Orlicz sequence spaces $l^{\varphi}$ generated by finite-valued Musielak-Orlicz function

$\varphi=\left(\varphi_{i}\right)_{i=1}^{\infty}$ which satisfies an additional condition $(+)$. In the case when $\varphi$ is an Orlicz function this formula coincides with formula (1). 
We say a Musielak-Orlicz function $\varphi=\left(\varphi_{i}\right)_{i=1}^{\infty}$ satisfies condition $(+)$ if for any $c>0$ and any $\varepsilon \in(0, c)$ there is $\delta>0$ such that $\varphi_{i}((1+$ $\delta) u) \leq c$ whenever $\varphi_{i}(u) \leq c-\varepsilon$ for $i=1,2, \ldots$ and $u \in \mathbf{R}_{+}$.

Assuming in this definition $c=1$ we obtain condition $\left({ }^{*}\right)$ defined by $A$. Kaminska in [7].

\section{RESULTS.}

To obtain main results we need to give some auxiliary lemmas.

Lemma 1 (see [4] and [6]). If $\varphi=\left(\varphi_{i}\right)_{i=1}^{\infty}$ is a finite-valued Musielak-Orlicz function i.e. all functions $\varphi_{i}$ are finite-valued and $\varphi$ satisfies the $\delta_{2}^{0}$-condition (equivalently, the $\delta_{2}$-condition), then $\|x\|_{\varphi}=1$ if and only if $I_{\varphi}(x)=1$.

Lemma 2. Let $\varphi=\left(\varphi_{i}\right)_{i=1}^{\infty}$ be a Musielak-Orlicz function satisfying condition $(+)$ and such that all $\varphi_{i}$ are finite-valued. For any sequence $\left(x^{k}\right)_{k=1}^{\infty}$ of elements in $S\left(l^{\varphi}\right)$ and $\delta>0$ there exist a subsequence $\left(y^{k}\right)_{k=1}^{\infty}$ of $\left(x^{k}\right)_{k=1}^{\infty}$ and a strictly increasing sequence $\left(i_{k}\right)_{k=1}^{\infty}$ of natural numbers such that

$$
\sum_{i=i_{k+1}+1}^{\infty} \varphi_{i}\left(y_{i}^{k}\right)<\delta \quad(k=1,2, \ldots)
$$

$$
\sum_{i=1}^{i_{k}} \varphi_{i}\left(y_{i}^{n}-y_{i}^{m}\right)<\delta / 2 \quad(k=1,2, \ldots ; m, n \geq k),
$$

$$
\sum_{i=i_{k}}^{i_{k+1}} \varphi_{i}\left(y_{i}^{n}\right)<\delta \quad(k=1,2, \ldots ; n \geq k),
$$

where $y_{i}^{k}$ denotes the $i$-th coordinate of $y^{k}$.

The lemma can be proved in the same way as Lemma 4 in [14]. Therefore, we omit the proof.

Lemma 3 (see [7]). Let $\varphi=\left(\varphi_{i}\right)_{i=1}^{\infty}$ be a finite-valued and satisfying the $\delta_{2}$-condition and condition $(+)$ Musielak-Orlicz function. 
For a given $\varepsilon>0$ and $c>0$ there exist $\delta=\delta(c, \varepsilon)>0$ such that $I_{\varphi}(x+y)<I_{\varphi}(x)+\varepsilon$ whenever $x, y \in l^{\varphi}, I_{\varphi}(x) \leq c$ and $I_{\varphi}(y)<\delta$.

Corollary 1. Under the assumption of Lemma 3 concerning $\varphi$ we have that for any $\eta>0$ there exists $\alpha>0$ such that $I_{\varphi}(x-y) \geq \alpha$ whenever $x, y \in l^{\varphi}, I_{\varphi}(x) \geq \eta, I_{\varphi}(y)<\eta / 2$.

Proof. Assume that $\varphi$ satisfies the assumptions and that the assertion from the corollary does not hold, i.e. there exists $\eta>0$ such that for any $\alpha>0$ there exist $x, y \in l^{\varphi}$ such that

$$
I_{\varphi}(x) \geq \eta, I_{\varphi}(y)<\frac{\eta}{2} \text { and } I_{\varphi}(x-y)<\alpha .
$$

Let $\delta>0$ be the number corresponding to $c=\varepsilon=\frac{\eta}{2}$ in Lemma 3 , and assume $\alpha=\delta$. Then we have

$$
I_{\varphi}(x)=I_{\varphi}(y+(x-y)) \leq I_{\varphi}(y)+\frac{\eta}{2}<\frac{\eta}{2}+\frac{\eta}{2}=\eta
$$

a contradiction. Therefore, the corollary is proved.

Now, we shall introduce a parameter $d_{\varphi}$ for finite-valued MusielakOrlicz functions $\varphi=\left(\varphi_{i}\right)_{i=1}^{\infty}$.

Define

$c(x, m, n)=\inf \left\{c>0: \sum_{i=n}^{n+m} \varphi_{i}\left(\frac{x_{i}}{c}\right) \leq \frac{1}{2}\right\} \quad\left(\forall x \in S\left(l^{\varphi}\right) ; m, n \in \mathbf{N}\right)$.

The sequence $(c(x, m, n))_{m=1}^{\infty}$ is nondecreasing for any $x \in S\left(l^{\varphi}\right)$ and $n \in \mathbf{N}$. Therefore, for any $x \in S\left(l^{\varphi}\right)$ and $n \in \mathbf{N}$, the limit

$$
d(x, n)=\lim _{m \rightarrow+\infty} c(x, m, n)
$$

exists. Moreover, we have $d(x, n) \geq c(x, m, n)$ for any $x \in S\left(l^{\varphi}\right)$ and $m, n \in \mathbf{N}$. Let

$$
d_{n}=\sup \left\{d(x, n): x \in S\left(l^{\varphi}\right)\right\} \quad(\forall n \in \mathbf{N})
$$


It is easy to see that $1 \leq d_{n} \leq 2$ for each $n \in \mathbf{N}$. Since the sequence $\left(d_{n}\right)_{n=1}^{\infty}$ is nonincreasing, we can define

$$
d_{\varphi}=\lim _{n \rightarrow+\infty} d_{n}
$$

Obviously, $1 \leq d_{\varphi} \leq 2$

Remark 1. Note that if we change only finite number of Orlicz functions $\varphi_{i}$ in the sequence $\varphi=\left(\varphi_{i}\right)_{i=1}^{\infty}$, the parameter $d_{\varphi}$ remains the same. Also the $\delta_{2}^{0}$-condition holds true for the Musielak-Orlicz function changed in such a way, whenever $\varphi$ satisfies the $\delta_{2}^{0}$-condition.

We are now in a position to give one of the main results of this paper.

Theorem 1. Let $\varphi=\left(\varphi_{i}\right)_{i=1}^{\infty}$ be a finite-valued Musielak-Orlicz function satisfying the $\delta_{2}^{0}$-condition and condition $(+)$. Then $D\left(l^{\varphi}\right)=$ $d_{\varphi}$.

Proof. First, we will prove that $D\left(l^{\varphi}\right) \geq d_{\varphi}$. For any $\varepsilon>0$ and each $n_{1} \in \mathbf{N}$ there exist $x^{1} \in S\left(l^{\varphi}\right)$ and $m_{1} \in \mathbf{N}$ such that

$$
c\left(x^{1}, m_{1}, n_{1}\right)>d\left(x^{1}, n_{1}\right)-\frac{\varepsilon}{4}>d_{n_{1}}-\frac{\varepsilon}{2} \geq d_{\varphi}-\frac{\varepsilon}{2},
$$

i.e.

$$
\sum_{i=n_{1}}^{n_{1}+n_{1}} \varphi_{i}\left(\frac{x_{i}^{1}}{d_{\varphi}-\frac{\varepsilon}{2}}\right) \geq \frac{1}{2} .
$$

Take $n_{2}=m_{1}+n_{1}+1$. There exists $x^{2} \in S\left(l^{\bullet}\right)$ and $m_{2}>m_{1}$ such that

i.e.

$$
c\left(x^{2}, m_{2}, n_{2}\right) \geq d_{\varphi}-\frac{\varepsilon}{2},
$$

$$
\sum_{i=n_{2}}^{n_{2}+m_{2}} \varphi_{i}\left(\frac{x_{i}^{2}}{d_{\varphi}-\frac{\varepsilon}{2}}\right) \geq \frac{1}{2} .
$$


Generally, putting $n_{k}=n_{k-1}+m_{k-1}+1$, there exist $x^{k} \in S\left(l^{\varphi}\right)$ and $m_{i+1}>m_{i}$ such that

$$
c\left(x^{k}, m_{k}, n_{k}\right)>d_{\varphi}-\frac{\varepsilon}{2}
$$

i.e.

$$
\sum_{i=n_{k}}^{n_{k}+m_{k}} \varphi_{i}\left(\frac{x_{i}^{k}}{d_{\varphi}-\frac{\varepsilon}{2}}\right) \geq \frac{1}{2} .
$$

Let $y^{k}=\left(y_{i}^{k}\right)_{i=1}^{\infty}$, where

$$
y_{i}^{k}= \begin{cases}x_{i}^{k} & \text { when } n_{k} \leq i \leq n_{k}+m_{k} \\ 0 & \text { otherwise }\end{cases}
$$

Define $z=\left(z^{k}\right)_{k=1}^{\infty}$, where $z^{k}=y^{k} /\left\|y^{k}\right\|_{\varphi}$. Then $z^{k} \in S\left(l^{\varphi}\right)$ and $\left\|y^{k}\right\|_{\varphi} \leq\left\|x^{k}\right\|_{\varphi}=1$. Therefore,

$$
\begin{aligned}
I_{\varphi}\left(\frac{z^{k}-z^{l}}{d_{\varphi}-\frac{\varepsilon}{2}}\right) & =\sum_{i=n_{k}}^{n_{k}+m_{k}} \varphi_{i}\left(\frac{x_{i}^{k}}{\left\|y^{k}\right\|_{\varphi}\left(d_{\varphi}-\frac{\varepsilon}{2}\right)}\right)+\sum_{i=n_{l}}^{n_{l}+m_{i}} \varphi_{i}\left(\frac{x_{i}^{l}}{\left\|y^{l}\right\|_{\varphi}\left(d_{\varphi}-\frac{\varepsilon}{2}\right)}\right) \\
& \geq \sum_{i=n_{k}}^{n_{k}+m_{k}} \varphi_{i}\left(\frac{x_{i}^{k}}{d_{\varphi}-\frac{\varepsilon}{2}}\right)+\sum_{i=n_{l}}^{n_{l}+m_{l}} \varphi_{i}\left(\frac{x_{i}^{l}}{d_{\varphi}-\frac{\varepsilon}{2}}\right) \geq \frac{1}{2}+\frac{1}{2}=1 .
\end{aligned}
$$

This means that $\left\|z^{k}-z^{l}\right\|_{\varphi} \geq d_{\varphi}-\frac{\varepsilon}{2}$, whence it follows immediately that $D\left(l^{\varphi}\right) \geq d_{\varphi}$.

We shall prove now that $D\left(l^{\varphi}\right) \leq d_{\varphi}$. Assume that this inequality does not hold. Then there exists a natural number $n$ such that $D\left(l^{\varphi}\right)>$ $d_{n}$. Denote $\eta=D\left(l^{\varphi}\right)-d_{n}$. By the definition of $D\left(l^{\varphi}\right)$ there exists a sequence $x=\left(x^{k}\right)_{k=1}^{\infty}$ such that

$$
\left\|x^{k}-x^{l}\right\|_{\varphi}>D\left(l^{\varphi}\right)-\frac{\eta}{3} \quad(\forall k, l \in \mathbf{N} ; k \neq l)
$$


Put $\varepsilon=(\eta / 3) / 2\left(d_{n}+\eta / 3\right)$. In view of Lemma 3 there exists $\delta \in(0, \varepsilon)$ such that

$$
I_{\varphi}(x+y)<I_{\varphi}(x)+\frac{\varepsilon}{2}
$$

whenever $I_{\varphi}(x) \leq 1$ and $I_{\varphi}(y)<\delta$.

In virtue of Lemma 2 we can choose a subsequence $\left(y^{k}\right)_{k=1}^{\infty}$ of $\left(x^{k}\right)_{k=1}^{\infty}$ satisfying conditions (i), (ii) and (iii) from this lemma with $\delta / 2$ instead of $\delta$.

For any $n_{1}, n_{2} \in \mathbf{N}, n \leq n_{1}<n_{2}$, and any $y \in S\left(l^{\varphi}\right)$, we have $d_{n} \geq c\left(y, n_{2}-n_{1}, n_{1}\right)$. Therefore,

$$
\begin{gathered}
\sum_{i=n_{1}}^{n_{2}} \varphi_{i}\left(\frac{y_{i}}{d_{n}+\frac{\eta}{3}}\right) \leq \sum_{i=n_{1}}^{n_{2}} \frac{d_{n}}{d_{n}+\frac{\eta}{3}} \varphi_{i}\left(\frac{y_{i}}{d_{n}}\right) \leq \\
\leq \frac{d_{n}}{d_{n}+\frac{\eta}{3}} \sum_{i=n_{1}}^{n_{2}} \varphi_{i}\left(\frac{y_{i}}{c\left(y, n_{1}, n_{2}-n_{1}\right)}\right) \leq \frac{d_{n}}{2\left(d_{n}+\frac{\eta}{3}\right)}=\frac{1}{2}-\varepsilon .
\end{gathered}
$$

For all natural numbers $k, l ; k<l$, we have

$$
I_{\varphi}\left(\frac{y^{k}-y^{l}}{d_{n}+\eta / 3}\right)=\left(\sum_{i=1}^{i_{k}}+\sum_{i=i_{k}+1}^{i_{k+1}}+\sum_{i=i_{k+1}+1}^{\infty}\right) \varphi_{i}\left(\frac{y_{i}^{k}-y_{i}^{l}}{d_{n}+\eta / 3}\right) .
$$

We may assume without loss of generality that $i_{k}>n$. In view of (ii) in Lemma 2 and the inequality $d_{n} \geq 1$, we get

$$
\sum_{i=1}^{i_{k}} \varphi_{i}\left(\frac{y_{i}^{k}-y_{i}^{l}}{d_{n}+\eta / 3}\right) \leq \sum_{i=1}^{i_{k}} \varphi_{i}\left(y_{i}^{k}-y_{i}^{l}\right)<\frac{\delta}{2} \leq \frac{\varepsilon}{2} .
$$

In virtue of (iii) in Lemma 2 and the inequality $d_{n} \geq 1$ we have




Moreover, by (15) we have

$$
\sum_{i=i_{k+1}}^{i_{k+1}} \varphi_{i}\left(\frac{y_{i}^{k}}{d_{n}+\eta / 3}\right) \leq \frac{1}{2}-\varepsilon
$$

Applying (14) with $\left(0, \ldots 0,-y_{i_{k}+1}^{k}, \ldots,-y_{i_{k+1}}^{k}, 0, \ldots\right)$ in place of $x$ and $\left(0, \ldots 0, y_{i_{k}+1}^{l}, \ldots, y_{i_{k+1}}^{l}, 0, \ldots\right)$ in place of $y$, we get

$$
\sum_{i=i_{k}+1}^{i_{k+1}} \varphi_{i}\left(\frac{y_{i}^{k}-y_{i}^{l}}{d_{n}+\eta / 3}\right)<\frac{1}{2}-\frac{\varepsilon}{2}
$$

We have by Lemma 2 (i) and (iii) with $\delta / 2$ instead of $\delta$ and $l=k+1$ that

$$
\sum_{i=i_{k+1}+1}^{\infty} \varphi_{i}\left(y_{i}^{j}\right)<\delta \quad(j=k, k+1),
$$

so applying again inequality (14), we get

$$
\sum_{i=i_{k+1}+1}^{\infty} \varphi_{i}\left(\frac{y_{i}^{k}-y_{i}^{l}}{d_{n}+\eta / 3}\right) \leq \sum_{i=i_{k+1}+1}^{\infty} \varphi_{i}\left(y_{i}^{k}-y_{i}^{l}\right)<\delta+\frac{\varepsilon}{2} .
$$

Combining now inequalities (17), (20) and (21), we get

$$
I_{\varphi}\left(\frac{y^{k}-y^{i}}{d_{n}+\eta / 3}\right) \leq\left(\frac{1}{2}-\frac{\varepsilon}{2}\right)+\left(\delta+\frac{\varepsilon}{2}\right)+\frac{\varepsilon}{2} \leq 1
$$

whence it follows that

$$
\left\|y^{k}-y^{l}\right\| \leq d_{n}+\eta / 3=D\left(l^{\varphi}\right)-\frac{2 \eta}{3},
$$

which contradicts (13), and consequently finishes the proof.

Theorem 2. If $\varphi=\left(\varphi_{i}\right)_{i=1}^{\infty}$ is a Musielak-Orlicz function which does not satisfy the $\delta_{2}^{0}$-condition, then $\Lambda\left(l^{\varphi}\right)=\frac{1}{2}$. 
Proof. It follows from the assumption that $l^{\varphi}$ contains an isomorphically isometric copy of $l^{\infty}$. In the case when all $\varphi_{i}$ are finite-valued this was proved by Kaminska in [6]. The proof can be repeated in the general case. Therefore,

$$
2=D\left(l^{\infty}\right) \leq D\left(l^{\varphi}\right) \leq 2
$$

whence we get $D\left(l^{\infty}\right)=2$, i.e. $\Lambda\left(l^{\varphi}\right)=\frac{1}{2}$, what completes the proof.

Corollary 1. Let $l^{\left(p_{i}\right)}$ be a Nakano space, where $l \leq p_{i}<+\infty$ for any $i \in \mathbf{N}$. Then:

$$
\begin{gathered}
\Lambda\left(l^{\left(p_{i}\right)}\right)=\frac{1}{2}, \quad \text { if } \limsup _{i \rightarrow+\infty} p_{i}=+\infty, \\
\Lambda\left(l^{\left(p_{i}\right)}\right)=2^{\frac{1}{p}} /\left(2+2^{\frac{1}{p}}\right), \quad \text { if } \limsup _{i \rightarrow+\infty} p_{i}<+\infty,
\end{gathered}
$$

where $p=\liminf _{i \rightarrow+\infty} p_{i}$.

Proof. The space $l^{\left(p_{i}\right)}$ is the Musielak-Orlicz space $l^{\varphi}$, where $\varphi=$ $\left(\varphi_{i}\right)_{i=1}^{\infty}$ is the Musielak-Orlicz function with $\varphi_{i}(u)=|u|^{p_{i}}$ for each $i \in \mathbf{N}$ and $u \in \mathbf{R}$. It is obvious that $\varphi$ satisfies the $\delta_{2}^{0}$-condition if and only if $\limsup p_{i}<+\infty$ and that the $\delta_{2}^{0}$-condition implies in this case $i \rightarrow+\infty$ condition $(+)$. Therefore, (22) follows inmediately from Theorem 2.

Assume now that $\limsup _{i \rightarrow+\infty} p_{i}<+\infty$ and define $p=\liminf _{i \rightarrow+\infty} p_{i}$.

Take an arbitrary $\varepsilon>0$. There exists $j \in \mathbf{N}$ such that $p_{i} \geq p-\varepsilon$ for any $i \geq j$.

Given $\alpha>0$ there exist $m \in \mathbf{N}$ and $x \in S\left(l^{\varphi}\right)$ such that $x_{i}=0$ for $i \leq n$ and $c(x, m, n) \geq d_{n}-\alpha / 2$. We have

$$
\sum_{i=n}^{n+m} \varphi_{i}\left(\frac{x_{i}}{d_{n}-\alpha}\right)=\sum_{i=n}^{n+m}\left(\frac{x_{i}}{d_{n}-\alpha}\right)^{p_{i}} \geq \sum_{i=1}^{n+m}\left(\frac{x_{i}}{c(x, m, n)-\alpha / 2}\right)^{p_{i}} \geq \frac{1}{2}
$$

whence 


$$
\sum_{i=n}^{\infty} \varphi_{i}\left(\frac{x_{i}}{d_{n}-\alpha}\right)=\sum_{i=1}^{\infty}\left(\frac{x_{i}}{d_{n}-\alpha}\right)^{p_{i}} \geq \frac{1}{2}
$$

Then we get for any $x \in S\left(l^{\varphi}\right)$ :

$$
\frac{1}{2} \leq \sum_{i=n}^{\infty}\left(\frac{x_{i}}{d_{n}-\alpha}\right)^{p_{i}}=\sum_{i=1}^{\infty} \frac{x^{p_{i}}}{\left(d_{n}-\alpha\right)^{p_{i}}} \leq \frac{1}{\min \left[\left(d_{n}-\alpha\right)^{p},\left(d_{n}-\alpha\right)^{p-\varepsilon}\right]}
$$

Hence, in view of the arbitrariness of $\varepsilon>0$, we get $d_{n}-\alpha \leq 2^{1 / p}$, whence $d_{\varphi} \leq 2^{1 / p}$ by the arbitrariness of $\alpha>0$.

Conversely, for any $\varepsilon>0$ there is an infinite subset $A$ of $\mathrm{N}$ such that $p_{i} \leq p+\varepsilon$ for any $i \in A$. Denote the sequence $\left(\varphi_{i}\right)_{i \in A}$ by $\Psi$. If $\varphi=\left(\varphi_{i}\right)_{i \geq 1}$ is a sequence of Orlicz functions and $\Psi=\left(\Psi_{j}\right)_{j \geq 1}$ is a subsequence of $\varphi$, then $d_{\Psi} \leq d_{\varphi}$. So, we can assume without loss of generality that $A=\mathbf{N}$ and $\Psi=\varphi$. Take any $m, n \in \mathbf{N}$ and $x \in S\left(l^{\varphi}\right)$ with supp $x=\left\{i \in \mathbf{N}: x_{i} \neq 0\right\} \subset\{n, n+1, \ldots, n+m\}$. We have

$$
\begin{aligned}
& \frac{1}{2}=\sum_{i=n}^{n+m} \varphi_{i}\left(\frac{x_{i}}{\underline{\underline{c(x, m, n)}}}\right)=\sum_{i=n}^{n+m} \frac{\left|x_{i}\right|^{p_{i}}}{(c(x, m, n))^{p_{i}}} \\
& \geq \frac{1}{[c(x, m, n)]^{p+\varepsilon}} \sum_{i=n}^{n+m}\left|x_{i}\right|^{p_{i}}=\frac{1}{[c(x, m, n)]^{p+\varepsilon}} .
\end{aligned}
$$

Hence it follows that $d_{\varphi} \geq 2^{1 / p}$ since $\varepsilon>0$ is arbitrary.

Note. The packing constant of $c_{0}$ is equal to $\frac{1}{2}$.

Proof. Taking the sequence

$$
\begin{gathered}
x^{1}=(1,0, \ldots), x^{2}=(-1,1,0, \ldots), \ldots, \\
x^{n}=(\underbrace{-1, \ldots,-1}_{(n-1) \text { times }}, 1,0, \ldots), \ldots,
\end{gathered}
$$


we have $x^{n} \in S\left(c_{0}\right)$ for any $n \in N$ and $\left\|x^{m}-x^{n}\right\|_{\infty}=2$ for any $m, n \in \mathbf{N}, m \neq n$. Therefore, $D\left(c_{0}\right)=2$, i.e. $\Lambda\left(c_{0}\right)=\frac{1}{2}$.

Theorem 3. If $\varphi=\left(\varphi_{i}\right)_{i=1}^{\infty}$ is a Musielak-Orlicz function such that all functions $\varphi_{i}$ are finite-valued then $\Lambda\left(h^{\varphi}\right)=\Lambda\left(l^{\varphi}\right)$.

Proof. Assume first that $\varphi$ satisfies the $\delta_{2}^{0}$-condition. Then $h^{\varphi}=$ $l^{\varphi}$ (see [5]), whence the desired equality follows.

Assume now that $\varphi$ does not satisfy the $\delta_{2}^{0}$-condition and take an arbitrary $\varepsilon>0$. Then there exists a closed subspace $l$ of $h^{\varphi}$ and a linear operator $P: c_{0} \stackrel{\text { onto }}{\longrightarrow} l$ such that

$$
\|x\|_{\infty} \leq\|P x\|_{\varphi} \leq(1+\varepsilon)\|x\|_{\infty} \quad\left(\forall x \in c_{0}\right)
$$

(see [1]). Consider the sequence $\left(x^{n}\right)_{n=1}^{\infty}$ defined in (24) and define a new sequence $\left(P x^{n} /\left\|P x^{n}\right\|_{\varphi}\right)_{i=1}^{\infty}$ in $l$. In view of (25) we have

$$
\left\|\frac{x^{i}}{\left\|P x^{i}\right\|_{\varphi}}-\frac{x^{j}}{\left\|P x^{j}\right\|_{\varphi}}\right\|_{\infty} \leq\left\|P\left(\frac{x^{i}}{\left\|P x^{i}\right\|_{\varphi}}\right)-P\left(\frac{x^{j}}{\left\|P x^{j}\right\|_{\varphi}}\right)\right\|,
$$

and

$$
\left|\frac{1}{\left\|P x^{i}\right\|_{\varphi}}-\frac{1}{\left\|p x^{j}\right\|_{\varphi}}\right| \leq 1-\frac{1}{1+\varepsilon}=\frac{\varepsilon}{1+\varepsilon} \leq \varepsilon .
$$

Applying (27), we get

$$
\begin{aligned}
\left\|\frac{x^{i}}{\left\|P x^{i}\right\|_{\varphi}}-\frac{x^{j}}{\left\|P x^{j}\right\|_{\varphi}}\right\|_{\infty} \geq & \left\|\frac{x^{i}-x^{j}}{\left\|P x^{i}\right\|_{\varphi}}\right\|_{\infty}-\left|\frac{1}{\left\|P x^{i}\right\|_{\varphi}}-\frac{1}{\left\|P x^{j}\right\|_{\varphi}}\right| \\
& \geq \frac{\left\|x^{i}-x^{j}\right\|_{\varphi}}{1+\varepsilon}-\varepsilon=\frac{\underline{2}}{1+\varepsilon}-\varepsilon
\end{aligned}
$$

Combining (26) and (28), we have

$$
\left\|P\left(\frac{x^{i}}{\left\|P x^{i}\right\|_{\varphi}}\right)-P\left(\frac{x^{i}}{\left\|P x^{j}\right\|_{\varphi}}\right)\right\|_{\varphi} \geq \frac{2}{1+\varepsilon}-\varepsilon .
$$


Since $\varepsilon>0$ is arbitrary it follows that $D\left(h^{\varphi}\right) \geq D(l)=2$. In view of the obvious inequality $D\left(h^{\varphi}\right) \leq 2$, we get $D\left(h^{\varphi}\right)=2$. The proof is finished.

Acknowledgements. The authors thank the referee for valuable comments and remarks.

The results of this paper were obtained in September 1989 when the first named author visited Harbin Institute of Technology in Harbin, China. He want to thank Prof. Wu Congxin for invitation and very good hospitality and the government of Harbin Institute of Technology for financial support.

\section{References}

[1] Alherk, G. and Hudzik, H. Copies of $l^{1}$ and $c_{0}$ in Musielak-Orlicz sequence spaces, Comment. Math. Univ. Carolinae, 35, 1 (1994), 9-19.

[2] Burlak, J., Rankin, R. and Robertson, A.R. The packing of spheres in the space $l_{p}$. Proc. Glasgow Math. Assoc. 4(1958), 22-25.

[3] Cleaver, C.E. Packing spheres in Orlicz spaces, Pacific J. Math. 65 (1976), 325-335.

[4] Denker, M. and Hudzik, H. Uniformly non-l(n) $l_{n}^{(1)}$ Musielak-Orlicz sequence spaces, Proc. Indian Acad. Sci. 101, 2(1991), 71-86.

[5] Hudzik, H. and Ye, Y. Support functionals and smoothness in Musielak-Orlicz sequence spaces, Comment. Math. Univ. Carolinae, 31, 4 (1990), 661-684.

[6] Kaminska, A. Flat Orlicz-Musielak sequence spaces. Bull. Acad. Polon. Sci. Math. 30, 7-8 (1982), 347-352.

[7] Kaminska, A. Uniform rotundity of Musielak-Orlicz sequence spaces. J. Approximation Theory 47 (4) (1986), 302-322.

[8] Kottman, C.A. Packing and reflexivity in Banach spaces. Trans. Amer. Math. Soc. 150 (1970), 565-576.

[9] Krasnoselskii, M.A. and Rutickii, Ya. B. Convex functions and Orlicz spaces. Groningen 1961 (translation). 
[10] Luxemburg, W.A.J. Banach function spaces, Thesis, Delfet 1955.

[11] Musielak, J. Orlicz spaces and modular spaces. Lecture Notes in Math. 1034 (1983).

[12] Wells, J. and Williams, L.R. Embeddings and extensions in analysis. Lecture Notes in Math. (1975).

[13] Wnuk, W. Orlicz spaces cannot be normed analogously to $L^{p}$-spaces. Indagationes Math. 46, 3 (1984), 357-359.

[14] Ye, Y. Packing spheres in Orlicz sequence spaces. Chinese Ann. Math. 4A, 4 (1983), 487-493 (in Chinese).

[15] Zaanen, A.C. Some remarks about the definition of an Orlicz space. Lecture Notes in Math. 945 (1982), 263-268.

Henryk Hudzik

Faculty of Mathematics and Informatics

A. Mickiewicz University

Foznan, Poland

Yining $\mathrm{Ye}$

Department of Mathematics

Harbin University of Sciences and Technology

Harbin, P.R. China
Congrin Wu

Department of Mathematics

Harbin Institute of Technology

Harbin, P.R. China

Recibido: 9 de octubre de 1992

Revisado: 27 de octubre de 1993 